Annals of Pure and Applied Mathematics

Vol. 16, No. 1, 2018, 1-6

ISSN: 2279-087X (P), 2279-0888(online)

Published on 1 January 2018

www.researchmathsci.org

DOI: http://dx.doi.org/10.22457/apam.v16n1al

Annals of

Pure and Applied

Mathematics

\title{
Results of Symmetric Reverse bi-derivations on Prime Rings
}

\author{
C.Jaya Subba Reddy, A.Sivakameshwara Kumar ${ }^{2}$ and B.Ramoorthy Reddy ${ }^{3}$ \\ ${ }^{1}$ Department of Mathematics, Sri Venkateswara University \\ Tirupati-517502, India. E-mail: cjsreddysvu@ gmail.com \\ ${ }^{2}$ Department of Mathematics, Rayalaseema University \\ Kurnool, Andhrapradesh, India. E-mail: kamesh1069@ gmail.com \\ ${ }^{3}$ Department of Mathematics, Sri Venkateswara University \\ Tirupati-517502, India. E-mail: ramoorthymaths@ gmail.com
}

Received 3 November 17; accepted 2 December 2017

Abstract. Let $R$ be a prime ring with $\operatorname{char} R \neq 2,3$ and let $d$ be trace of a nonzero symmetric reverse bi-derivation $D(.,$.$) . For a fixed element a$ of $R$ with $d(a) \neq 0$, if the identity $d(x) a d(x)=0$ holds for all $x \in R$, then $a \in Z$.

Keywords: Derivation, reverse derivation, symmetric, symmetric bi-derivation, symmetric reverse bi-derivation, prime rings, trace.

\section{AMS Mathematics Subject Classification (2010): 13D03}

\section{Introduction}

The concept of a symmetric bi-derivation has been introduced by Maksa in [6]. In [9], Vukman has proved some results concerning symmetric bi-derivation on prime and semiprime rings. Yenigul and Argac [10] studied ideals and symmetric bi-derivations of prime and semiprime rings. Reddy et al. [5] studied symmetric reverse bi-derivations on prime rings. Sapanci et al. [8] studied few results of symmetric bi-derivation on prime rings. In this paper, we extended some results of symmetric reverse bi-derivations on prime rings.

\section{Preliminaries}

Throughout this paper $R$, will be denoted an associative ring with the center $Z(R)$. Recall that a ring $R$ is called prime if for any $a, b \in R, a R b=(0)$ implies that either $a=0$ or $b=0$. For any $x, y \in R$, the symbol $[x, y]$ stands for commutator $x y-y x$. An additive mapping $d: R \rightarrow R$ is said to be a derivation if $d(x y)=d(x) y+x d(y)$, for all $x, y \in R$. An additive mapping $d: R \rightarrow R$ is said to be a reverse derivation if $\mathrm{d}(x y)=d(y) x+$ $y d(x)$, for all $x, y \in R$. A mapping $D(.,):. R \times R \rightarrow R$ is said to be symmetric if $D(x, y)=D(y, x)$, for all $x, y \in R$. A mapping $d: R \rightarrow R$ is called the trace of $D(.,$.$) if$ $d(x)=D(x, x)$, for all $x \in R$. It is obvious that if $D(.,$.$) is bi-additive (i.e., additive in$ both arguments), then the trace $d$ of $D(.,$.$) satisfies the identity d(x+y)=d(x)+$ 
C.Jaya Subba Reddy, A.Sivakameshwara Kumar and B.Ramoorthy Reddy

$d(y)+2 D(x, y)$, for all $x, y \in R$. If $D(.,$.$) is bi-additive and satisfies the identities$ $D(x y, z)=D(x, z) y+x D(y, z)$ and

$$
D(x, y z)=D(x, y) z+y D(x, z) \text {, for all } x, y, z \in R .
$$

Then $D(.,$.$) is called a symmetric bi-derivation. If D(.,$.$) is reverse bi-additive and$ satisfies the identity $D(x y, z)=D(y, z) x+y D(x, z)$

and $D(x, y z)=D(x, z) y+z D(x, y)$, for all $x, y, z \in R$. Then $D(.,$.$) is called a$ symmetric reverse bi-derivation. We shall make use of commutator identities; $[x, y z]=$ $[x, y] z+y[x, z]$ and $[x y, z]=[x, z] y+x[y, z]$, for all $x, y, z \in R$.

Lemma 1. Let $R$ be a prime ring with $\operatorname{char} R \neq 2, D(.,$.$) a symmetric reverse bi$ derivation and $d$ the trace of $D(.,$.$) . If U$ is a nonzero ideal of $R$ such that $a d(U)=0$ (or, $d(U) a=0)$, then $a=0$ or $d=0$.

Proof: Given that $\operatorname{ad}(U)=0$, for some nonzero ideal $U$.

By linearizing above equation, we get

$a d(u+v)=0$, for all $u, v \in U$.

$a d(u)+a d(v)+a 2 D(u, v)=0$

Since $d(u)=d(v)=0$ and $\operatorname{char} R \neq 2$, then

$a D(u, v)=0$, for all $u, v \in U$.

Replacing $v$ by $u v$ in above equation, we get

$a D(u, u v)=0$

$a(D(u, v) u+v D(u, u))=0$

$a D(u, v) u+a v D(u, u)=0$

$\operatorname{avd}(u)=0$

$\operatorname{aRd}(u)=0$

Since $R$ is a prime ring, then $a=0$ or $d=0$.

Lemma 2. [1, Theorem 3.1.3] Let $R$ be a prime ring with char $R \neq 2, D(.,$.$) a symmetric$ bi-derivation and $d$ the trace of $D(.,$.$) . For a fixed element a \in R$, we have

(i) If $[a, d(x)]=0$, for all $x \in R$, then $a \in Z$ or $d=0$.

(ii) If $[a, d(x)] \in Z$, for all $x \in R$ and for nonzero trace $d$ with $d(a) \neq 0$, then $a \in Z$.

Lemma 3. Let $R$ be a prime ring and let $a, b, c \in R$. If $a x b=c x a$ for all $x \in R$, then $a=0$ or $b=c$.

Proof: Given that $a x b=c x a$, for all $x \in R$.

Replacing $x$ by $x a y$ in above equation, we get

$a x a y b=$ cxaya.

But $a y b=c y a$ and $c x a=a x b$ then, we get

axcya $=$ axbya

axcya - axbya $=0$

$a x(c-b) y a=0$

Since $R$ is a prime ring, then $a=0$ or $b=c$.

Lemma 4. Let $R$ be a prime ring with $\operatorname{char} R \neq 2$ and let $d_{1}$ and $d_{2}$ be traces of symmetric reverse bi-derivations $D_{1}(.,$.$) and D_{2}(.,$.$) respectively. If the identity$ 
Results of Symmetric Reverse bi-derivations on Prime Rings

$d_{1}(x) d_{2}(y)=d_{2}(x) d_{1}(y)$ holds and $d_{1} \neq 0$, then there exists $\lambda \in C$ such that $d_{2}(x)=$ $\lambda d_{1}(x)$.

Proof: Given $d_{1}(x) d_{2}(y)=d_{2}(x) d_{1}(y)$, for all $x, y, z \in R$.

Replacing $y$ by $z+y$ in equation (1), we get

$$
\begin{gathered}
d_{1}(x) d_{2}(z+y)=d_{2}(x) d_{1}(z+y) \\
d_{1}(x) d_{2}(z)+d_{1}(x) d_{2}(y)+d_{1}(x) 2 D_{2}(z, y) \\
=d_{2}(x) d_{1}(z)+d_{2}(x) d_{1}(y)+d_{2}(x) 2 D_{1}(z, y) \\
d_{1}(x) D_{2}(z, y)=d_{2}(x) D_{1}(z, y)
\end{gathered}
$$

Replacing $z$ by $y z$ in equation (2) leads to the identity

$d_{1}(x) D_{2}(y z, y)=d_{2}(x) D_{1}(y z, y)$

$d_{1}(x) z D_{2}(y, y)+d_{1}(x) D_{2}(z, y) y=d_{2}(x) z D_{1}(y, y)+d_{2}(x) D_{1}(z, y) y$

$d_{1}(x) z d_{2}(y)=d_{2}(x) z d_{1}(y)$

Again replacing $y$ by $x$ in equation (3), we get

$d_{1}(x) z d_{2}(x)=d_{2}(x) z d_{1}(x)$

Thus if $d_{1}(x) \neq 0$, then by (4) and [4, Corollary to Lemma 1.3.2] we have $d_{2}(x)=\lambda$ $(x) d_{1}(x)$ for some $\lambda(x) \in C$. Hence if $d_{1}(x) \neq 0$ and $d_{1}(y) \neq 0$, then $(\lambda(y)-\lambda$ $(x)) d_{1}(x) z d_{1}(y)=0$ by equation (3). Since $R$ is prime, it follows from Lemma 1 that $\lambda(x)=\lambda(y)$.This shows that there exists $\lambda \in C$ such that $d_{2}(x)=\lambda d_{1}(x)$ under the condition $d_{1}(x) \neq 0$. On the other hand, assume that $d_{1}(x)=0$. Since $d_{1} \neq 0$ and $R$ is a prime, it follows from equation (3) that $d_{2}(x)=0$ as well. Thus $d_{2}(x)=\lambda d_{1}(x)$. This completes the proof.

Theorem 1. Let $R$ be a prime ring with $\operatorname{char} R \neq 2$ and let $d_{1}(\neq 0), d_{2}, d_{3}, d_{4}(\neq 0)$ be traces of symmetric reverse bi-derivations $D_{1}(.,),. D_{2}(.,),. D_{3}(.,$.$) and D_{4}(.,$. respectively. If the identity $d_{1}(x) d_{2}(y)=d_{3}(x) d_{4}(y)$, holds for all $x, y \in R$, then there exists $\lambda \in C$ such that $d_{2}(x)=\lambda d_{4}(x)$ and $d_{3}(x)=\lambda d_{1}(x)$.

Proof: Given $d_{1}(x) d_{2}(y)=d_{3}(x) d_{4}(y)$, for all $x, y \in R$.

Replacing $y$ by $z+y$ in equation (5), we get

$$
\begin{gathered}
d_{1}(x) d_{2}(z+y)=d_{3}(x) d_{4}(z+y) \\
d_{1}(x) d_{2}(z)+d_{1}(x) d_{2}(y)+d_{1}(x) 2 D_{2}(z, y) \\
=d_{3}(x) d_{4}(z)+d_{3}(x) d_{4}(y)+d_{3}(x) 2 D_{4}(z, y) \\
d_{1}(x) D_{2}(z, y)=d_{3}(x) D_{4}(z, y)
\end{gathered}
$$

Replacing $z$ by $y z$ in equation (6) and using equation (6) leads to the identity

$$
\begin{aligned}
& d_{1}(x) D_{2}(y z, y)=d_{3}(x) D_{4}(y z, y) \\
& d_{1}(x) z D_{2}(y, y)+d_{1}(x) D_{2}(z, y) y=d_{3}(x) z D_{4}(y, y)+d_{3}(x) D_{4}(z, y) y \\
& d_{1}(x) z d_{2}(y)=d_{3}(x) z d_{4}(y)
\end{aligned}
$$

It follows from replacing $z$ by $z d_{4}(w)$ in equation (7), and using equation (7), we get $d_{1}(x) z d_{4}(w) d_{2}(y)=d_{3}(x) z d_{4}(w) d_{4}(y)=d_{1}(x) z d_{2}(w) d_{4}(y)$

So that $d_{1}(x) z\left(d_{4}(w) d_{2}(y)-d_{2}(w) d_{4}(y)\right)=0$, for all $x, y, z, w \in R$.

Since $d_{1} \neq 0$ and $R$ is a prime, it follows that $d_{4}(w) d_{2}(y)=d_{2}(w) d_{4}(y)$. Applying Lemma 4, there exists $\lambda \in C$ such that $d_{2}(y)=\lambda d_{4}(y)$, Which implies from equation (7) that $\left(\lambda d_{1}(x)-d_{3}(x)\right) z d_{4}(y)=0$ so that $d_{3}(x)=\lambda d_{1}(x)$. This completes the proof. 
C.Jaya Subba Reddy, A.Sivakameshwara Kumar and B.Ramoorthy Reddy

Theorem 2. Let $R$ be a prime ring with $\operatorname{char} R \neq 2,3$ and let $d$ be trace of a nonzero symmetric reverse bi-derivations $D(.,$.$) . For a fixed element a$ of $R$ with $d(a) \neq 0$, if the identity $d(x) a d(x)=0$ holds for all $x \in R$, then $a \in Z$.

Proof: Given $d(x) a d(x)=0$, for all $x \in R, a \in Z$.

By linearizing $x$ by $x+y$ in equation (8) and using equation (8), we get

$$
\begin{aligned}
& d(x+y) a d(x+y)=0 \\
& \begin{aligned}
&(d(x)+d(y)+2 D(x, y)) a(d(x)+d(y)+2 D(x, y))=0 \\
& d(x) a d(x)+ d(y) a d(x)+2 D(x, y) a d(x)+d(x) a d(y)+d(y) a d(y) \\
&+2 D(x, y) a d(y)+d(x) a 2 D(x, y)+d(y) a 2 D(x, y) \\
&+2 D(x, y) a 2 D(x, y)=0
\end{aligned}
\end{aligned}
$$

$d(x) a d(y)+d(y) a d(x)+2 d(x) a D(x, y)+2 d(y) a D(x, y)+2 D(x, y) a d(x)+$ $2 D(x, y) a d(y)+4 D(x, y) a D(x, y)=0$, for all $x, y \in R$.

Substituting $x$ for $-x$ in equation (9), we get

$$
\begin{aligned}
& d(-x) a d(y)+d(y) a d(-x)+2 d(-x) a D(-x, y)+2 d(y) a D(-x, y)+ \\
& 2 D(-x, y) a d(-x)+2 D(-x, y) a d(y)+4 D(-x, y) a D(-x, y)=0 \\
& d(x) a d(y)-2 d(x) a D(x, y)+d(y) a d(x)-2 d(y) a D(x, y)-2 D(x, y) a d(x)- \\
& 2 D(x, y) a d(y)+4 D(x, y) a D(x, y)=0
\end{aligned}
$$

By adding equations (9) and (10), we get

$$
\begin{aligned}
& 2 d(x) a d(y)+2 d(y) a d(x)+8 D(x, y) a D(x, y)=0 \\
& 2(d(x) a d(y)+d(y) a d(x)+4 D(x, y) a D(x, y))=0
\end{aligned}
$$

Since $R$ is a char $R \neq 2$ then, we get

$d(x) a d(y)+d(y) a d(x)+4 D(x, y) a D(x, y)=0$

Now we replacing $x$ by $x+y$ in equation (11) and expand it, and then we use equations

(8), (11) and the fact that char $R \neq 2$ then, we get

$$
\begin{aligned}
& d(x+y) a d(y)+d(y) a d(x+y)+4 D(x+y, y) a D(x+y, y)=0 \\
& (d(x)+d(y)+2 D(x, y)) a d(y)+d(y) a(d(x)+d(y)+2 D(x, y))+4(D(x, y)+ \\
& D(y, y)) a(D(x, y)+D(y, y))=0 \\
& d(x) a d(y)+d(y) a d(y)+2 D(x, y) a d(y)+d(y) a d(x)+d(y) a d(y)+ \\
& 2 d(y) a D(x, y)+4 D(x, y) a D(x, y)+4 D(x, y) a D(y, y)+4 D(y, y) a D(x, y)+ \\
& 4 D(y, y) a D(y, y)=0 \\
& d(x) a d(y)+2 D(x, y) a d(y)+d(y) a d(x)+2 d(y) a D(x, y)+4 D(x, y) a D(x, y)+ \\
& 4 D(x, y) a d(y)+4 d(y) a D(x, y)+4 d(y) a d(y)=0 \\
& d(x) a d(y)+d(y) a d(x)+4 D(x, y) a D(x, y)+2 D(x, y) a d(y)+2 d(y) a D(x, y)+ \\
& 4 d(y) a D(x, y)+4 D(x, y) a d(y)=0 \\
& 2 D(x, y) a d(y)+2 d(y) a D(x, y)+4 d(y) a D(x, y)+4 D(x, y) a d(y)=0 \\
& \text { Since } R \text { is a charR } \neq 2 \text { then, we get } \\
& D(x, y) a d(y)+d(y) a D(x, y)+2 d(y) a D(x, y)+2 D(x, y) a d(y)=0 \\
& 3 D(x, y) a d(y)+3 d(y) a D(x, y)=0 \\
& D(x, y) a d(y)+d(y) a D(x, y)=0
\end{aligned}
$$

Replacing $y$ by $x+y$ in equation (12) then, we get

$D(x, x+y) a d(x+y)+d(x+y) a D(x, x+y)=0$ 
Results of Symmetric Reverse bi-derivations on Prime Rings

$$
\begin{aligned}
& (D(x, x)+D(x, y)) a(d(x)+d(y)+2 D(x, y))+ \\
& (d(x)+d(y)+2 D(x, y)) a(D(x, x)+D(x, y))=0 \\
& d(x) a d(x)+d(x) a d(y)+2 d(x) a D(x, y)+D(x, y) a d(x)+D(x, y) a d(y)+ \\
& 2 D(x, y) a D(x, y)+d(x) a d(x)+d(x) a D(x, y)+d(y) a d(x)+d(y) a D(x, y)+ \\
& 2 D(x, y) a d(x)+2 D(x, y) a D(x, y)=0 \\
& d(x) a d(y)+d(y) a d(x)+4 D(x, y) a D(x, y)+D(x, y) a d(y)+d(y) a D(x, y)+ \\
& 3 D(x, y) a d(x)+3 d(x) a D(x, y)=0 \\
& \text { Using equations }(8),(11),(12) \text { and the fact that char } R \neq 3, \text { we get } \\
& D(x, y) a d(x)+d(x) a D(x, y)=0
\end{aligned}
$$

Replacing $y$ by $z y$ in equation (13), and using equation (13), we get

$D(x, z y) a d(x)+d(x) a D(x, z y)=0$

$y D(x, z) a d(x)+D(x, y) z a d(x)+d(x) a y D(x, z)+d(x) a D(x, y) z=0$

$y D(x, z) a d(x)+D(x, y) z a d(x)+d(x) a y D(x, z)-D(x, y) a d(x) z=0$

$y D(x, z) a d(x)+D(x, y)[\operatorname{zad}(x)-a d(x) z)]+d(x) a y D(x, z)=0$

$y D(x, z) a d(x)+D(x, y)[z, a d(x)]+d(x) a y D(x, z)=0$

$-y d(x) a D(x, z)+D(x, y)[z, a d(x)]+d(x) a y D(x, z)=0$

$[d(x) a, y] D(x, z)+D(x, y)[z, a d(x)]=0$

$D(x, y)[z, \operatorname{ad}(x)]=-[d(x) a, y] D(x, z)$

$D(x, y)[z, a d(x)]=[y, d(x) a] D(x, z)$

Interchanging $x$ to $y$, and $y$ to $x$ and applying symmetric if $D(x, y)=D(y, x)$

$D(y, x)[z, a d(y)]=[x, d(y) a] D(y, z)$

$D(x, y)[z, a d(y)]=[x, d(y) a] D(z, y)$

Replacing $x$ by $w x$ in equation (14) and using equation (14) again, we get

$D(w x, y)[z, a d(y)]=[w x, d(y) a] D(z, y)$

$x D(w, y)[z, a d(y)]+D(x, y) w[z, a d(y)]=w[x, d(y) a] D(z, y)+[w, d(y) a] x D(z, y)$

$x D(w, y)[z, a d(y)]+D(x, y) w[z, a d(y)]=w D(x, y)[z, a d(y)]+[w, d(y) a] x D(z, y)$

Replacing $x$ to $w$ then, we get

$$
\begin{aligned}
& w D(w, y)[z, a d(y)]+D(w, y) w[z, a d(y)] \\
& \quad=w D(w, y)[z, \operatorname{ad}(y)]+[w, d(y) a] w D(z, y)
\end{aligned}
$$

$D(w, y) w[z, a d(y)]=[w, d(y) a] w D(z, y)$

Replacing $z$ to $w$ in equation (15) then, we get

$D(w, y) w[w, \operatorname{ad}(y)]=[w, d(y) a] w D(w, y)$

Replacing $w$ to $x$ then, we get

$D(x, y) x[x, \operatorname{ad}(y)]=[x, d(y) a] x D(x, y)$

It follows from Lemma 3 that $D(x, y)=0$ or $[x, a d(y)]=[x, d(y) a]$. In other words, $R$ is the union of its subsets $A=\{x \in R / D(x, y)=0$ for all $y \in R\}$ and $B=\{x \in$ $R /[x, \operatorname{ad}(y)-d(y) a]=0$ for all $y \in R\}$. Note that $A$ and $B$ are additive subgroups of $R$. Since $R$ cannot be written as the union of $A$ and $B$, it follows that $A=R$ or $B=R$. So from the hypothesis that $R=B$. This implies that $[a, d(y)] \in Z$ for all $y \in R$. By Lemma 2(ii), we know that $a \in Z$. This completes the proof.

\section{REFERENCES}

1. N.Argac, On prime rings with derivation, Ph.D. Thesis, Ege university,1992.

2. M.Bresar, A note on derivations, Math. J. Okayama Univ., 32 (1990) 83-88. 
C.Jaya Subba Reddy, A.Sivakameshwara Kumar and B.Ramoorthy Reddy

3. M.Bresar, Centralizing mappings and derivations in prime rings, J. Algebra, 156 (1993) 385-394.

4. I.N. Herstein, Rings with involution, Univ. of Chicago Press, Chicago, 1969.

5. C.Jaya Subba Reddy, Symmetric reverse bi-derivations on prime rings, Research J. Pharm. and Tech, 9(9) (2016) 1496-1500.

6. Gy.Maksa, On the trace of symmetric bi-derivations, C.R. Math. Rep. Sci. Canada, 9 (1987) 303-307.

7. J.Mayne, Centralizing mappings of prime rings, Canadian Math. Bull, 27(1) (1984) 122-126.

8. M. Sapanci, M.A.Ozturk and Y.B.Jun, Symmetric bi-derivations on prime rings, East Asian Math .J, 14(1) (1999) 105-109.

9. J.Vukman, Symmetric bi-derivations on prime ring and semi-prime rings, Aeuationes Mathematics, 38 (1989) 245-254.

10. M.S.Yenigul and N.Argac, Ideals and symmetric bi-derivations of prime and semiprime rings, Math. J. Okayama Univ, 35 (1993) 189-192. 